\title{
Advances in Imaging Reactive Oxygen Species
}

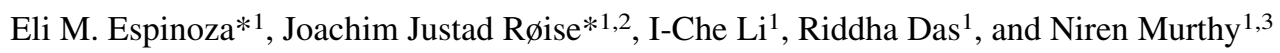 \\ ${ }^{I}$ Department of Bioengineering, University of California, Berkeley, California; ${ }^{2}$ Department of Chemistry, University of California, \\ Berkeley, California; and ${ }^{3}$ Innovative Genomics Institute, Berkeley, California
}

Reactive oxygen species (ROS) play a pivotal role in many cellular processes and can be either beneficial or harmful. The design of ROS-sensitive fluorophores has allowed for imaging of specific activity and has helped elucidate mechanisms of action for ROS. Understanding the oxidative role of ROS in the many roles it plays allows us to understand the human body. This review provides a concise overview of modern advances in the field of ROS imaging. Indeed, much has been learned about the role of ROS throughout the years; however, it has recently been shown that using nanoparticles, rather than individual small organic fluorophores, for ROS imaging can further our understanding of ROS.

Key Words: animal imaging; molecular imaging; ROS; chromophores; imaging; radicals

J Nucl Med 2021; 62:457-461

DOI: 10.2967/jnumed.120.245415

\section{$\mathbf{I}_{\mathrm{n}}$}

nature, reactive oxygen species (ROS) are unavoidable byproducts of aerobic metabolism. These oxygen-containing chemical species include hydrogen peroxide $\left(\mathrm{H}_{2} \mathrm{O}_{2}\right)$, singlet oxygen $\left({ }^{1} \mathrm{O}_{2}\right)$, the hydroxyl radical $\left({ }^{\bullet} \mathrm{OH}\right)$, peroxides $\left(\mathrm{O}_{2}^{2-}\right)$, and superoxides $\left(\mathrm{O}_{2}{ }^{\bullet-}\right)$. Additionally, reactive nitrogen species (RNS), such as nitric oxide (NO), nitric dioxide $\left(\mathrm{NO}_{2}\right)$, and peroxynitrite $\left(\mathrm{OONO}^{-}\right)$, also fall under the category of reactive species. The presence of ROS in the body is vital to functions ranging from killing foreign microbes to playing pivotal roles in cell-signaling pathways (1); ROS has also become a biomarker for oxidative stress caused by diseases such as Alzheimer disease, Parkinson disease, atherosclerosis, cancer, and depression, among others (2).

Because of their involvement in a myriad of diseases, ROS probes for both in vivo and in vitro detection are vital tools for clinical diagnostics. Although many probes have been developed, several problems need to be addressed to successfully yield a multipotent ROS probe. Some of the considerations in designing in situ ROS fluorescent probes include water solubility, aggregation, quantum yields, singlet excited-state lifetime, and excitation wavelength, which determines tissue penetration. Additionally, biologic considerations such as cell permeability, ROS selectivity, and reaction rates are important for meaningful detection. Because of the complex cellular environments in which ROS are monitored,

Received Oct. 2, 2020; revision accepted Dec. 8, 2020.

For correspondence or reprints contact: Niren Murthy, University of California, Berkeley, 284 Hearst Memorial Mining Building, Berkeley, CA 94720.

E-mail: nmurthy@berkeley.edu

${ }^{*}$ Contributed equally to this work.

Published online Dec. 31, 2020.

COPYRIGHT (C 2021 by the Society of Nuclear Medicine and Molecular Imaging. numerous diverse probes have been developed. In this review, we will focus on recent developments in the field of ROS imaging.

\section{REDUCED DYES FOR ROS IMAGING}

A common strategy to trigger a fluorescent readout in response to ROS is the use of a nonfluorescent reduced dye that, when oxidized by ROS, yields a fluorescent product. This strategy has been widely applied for decades, with compounds such as scopoletin being used to detect $\mathrm{H}_{2} \mathrm{O}_{2}$ in the presence of peroxidase in the 1950s (3). Reduced dyes are particularly attractive for ROS detection because they often can be generated from commercially available fluorescent dyes, allowing for trivial synthesis of a wide range of reduced dyes that can be used to detect and image ROS. This section will cover the reduced dyes that are most commonly used for ROS imaging.

\section{Hydrocyanines}

Since their discovery in 2009 (4), hydrocyanine probes have been used for detection of superoxide and hydroxyl ROS both in vitro and in vivo $(4,5)$, with detection limits down to nanomolar concentrations. A wide range of hydrocyanines with desired properties can be prepared in a single synthetic step, making them accessible even to labs with limited synthetic infrastructures.

Hydrocyanines were first discovered by Kundu et al. (4) in 2009 by reducing commercially available cyanine dyes with $\mathrm{NaBH}_{4}$. The hydrocyanine hydro-Cy3 successfully imaged ROS in rat aortic smooth muscle cells after treatment with the angiotensin II peptide, a system that mimics the development of atherosclerosis and hypertension. In addition, hydro-Cy7, a near-infrared (NIR) dye, was used to image ROS in mice during a lipopolysaccharidemediated inflammatory response. In the years following, hydrocyanines were extensively investigated for ROS imaging, most of which were covered in a previous review (5). We will therefore focus on recent developments in the field of hydrocyanines in the past 3 years.

Although hydrocyanines have many uses, they do have some limiting factors. These limitations include high autooxidation, low Stokes shifts, and low solubility, as well as their product cyanine dyes' lability to ROS (6). A strategy for reducing some of these shortcomings was published by Maity et al. (6), who introduced a new class of thiophene-bridged hydrocyanines (Fig. 1A). Thiophenebridged hydrocyanines showed superior stability to autooxidation, with $89 \%$ of the probe remaining after a 48 -h incubation in phosphatebuffered saline, compared with $42 \%$ for hydro-Cy5 (Fig. 1B). After oxidation, THBC generated a fluorescent product with significant stability toward ROS-mediated degradation (Fig. 1C). However, despite their promise, THBC derivatives have not been thoroughly studied, partly because of their relatively complex synthesis compared with regular hydrocyanines. Regardless, the increase in chemical stability and the improved photophysical 


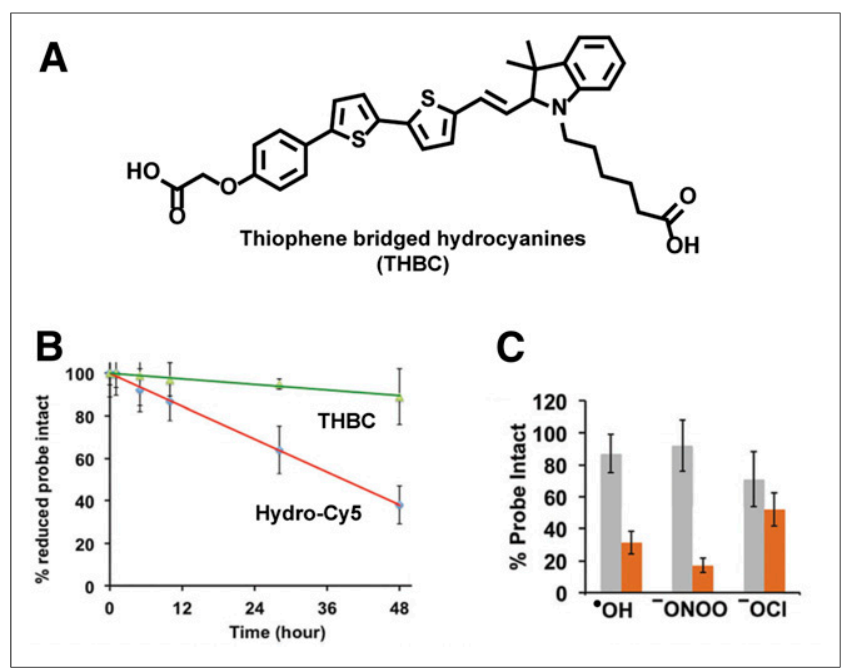

FIGURE 1. Modification of hydrocyanines for increased stability and in vivo sensitivity. (A) Structure of recently reported thiophene-bridged hydrocyanines (THBC). (B) Increase in stability of THBC toward autooxidation in phosphate-buffered saline. (C) Increase in stability of active thiophene-bridged cyanines (gray) toward ROS-dependent degradation, compared with Сy3 (orange). Graph represents percentage of probe intact after incubation with $250 \mu \mathrm{M}$ of indicated ROS. (Reprinted from Maity et al. (6).)

properties yield a promising scaffold that should be further investigated.

Because hydrocyanines are well established in their use for ROS detection, recent advances in the field have focused on building multifunctional systems to increase selectivity and introduce additional functions. For example, Al-Karmi et al. (7) introduced an ${ }^{18} \mathrm{~F}$ label on IR780, allowing for PET-based biodistribution studies of hydrocyanine probes. Andina et al. (8) used a modular design of a reported dye connected to hydro-Cy5 via 2 complementary peptide nucleic acid moieties for measuring extracellular ROS. The use of a reporter dye allowed them to account for distribution differences by using a ratio-based readout. In a study by Zhang et al. (9), glioblastomas were selectively imaged by conjugating hydro-Cy5 to the integrin $\alpha_{\mathrm{v}} \beta_{3}$-targeting peptide Arg-Trp-(D-Arg)-Asn-Arg. Using this strategy, tumors could be imaged selectively in the presence of other inflammatory tissues.

\section{Xanthene Probes}

Xanthene dyes are some of the most frequently used probes for ROS detection, with reduced derivatives of fluorescein and rhodamine being the most abundant. They can easily be prepared through direct reduction of their oxidized derivatives. Xanthene dyes were initially used as $\mathrm{H}_{2} \mathrm{O}_{2}$-selective probes but have been shown to react readily with hydroxyl and peroxyl radicals and with several RNS (10). This characteristic has reduced their practicality in favor of more selective probes, although they are still frequently used.

Although fluorescein and rhodamine-based dyes are heavily used for ROS detection, they do not possess any specificity toward the ROS source. One way to achieve organelle specificity was explored by Zhang et al. (11), who used silicon-rhodamine-based NIR fluorescent probes to target lysosomal ROS. These probes showed high specificity toward the highly reactive ROS, HClO, $\mathrm{HO}^{\bullet}$ and $\mathrm{ONOO}^{*}$ allowing for imaging of ROS in the lysosomes of cancer cells. This strategy was further explored by Wang et al., when they created a NIR probe for in vivo imaging of $\mathrm{HClO} / \mathrm{ONOO}$ in an idiopathic pulmonary fibrosis mouse model (12).

\section{ROS-SENSITIVE FUNCTIONAL GROUPS}

Several ROS probes possess a protecting or quenching group that is cleaved on ROS exposure. These probes depend largely on the nucleophilic properties of ROS, such as $\mathrm{H}_{2} \mathrm{O}_{2}$ and superoxides. This mechanism of action allows for ROS specificities that are complementary to reduced dyes. The main classes that will be covered in this section are sulfonyls, arylboronates, and phosphinates.

\section{Sulfonyls}

Sulfonyl-protected dyes were developed to battle shortcomings associated with other protecting groups such as acetyls, which show significant deprotection from hydrolysis in addition to perhydrolysis (13). The superoxide-selective sulfonyl probes were introduced when Maeda et al. (14) developed a diprotected fluorescein analog, although the observed selectivity depended on the sulfonyl substituent. Further studies were done to eliminate the impact of side reactions by modifying the leaving group. In particular, the triflate-protected probe HKSOX-1 showed excellent resistance to thiol-mediated cleavage, thus allowing for selective detection of superoxide in zebrafish embryos (15). Sulfonyls are still being developed for use in selective superoxide detection, with recent applications including superoxide detection in mitochondria (16) and the use of 2-photon excitation microscopy (TPEF) probes (17).

\section{Arylboronates}

Arylboronic acids have long been known for their reactivity toward $\mathrm{H}_{2} \mathrm{O}_{2}$, in which addition of peroxide to the boron leads to an aryl-group migration and subsequent phenol formation (18). Boronic acids mask the fluorescence of fluorophores, yielding $\mathrm{H}_{2} \mathrm{O}_{2}$-responsive probes. This method was pioneered by the Chang lab (19) when it synthesized a diboronic acid-modified fluorescein that selectively reacted with $\mathrm{H}_{2} \mathrm{O}_{2}$ in the presence of other ROS. After this seminal study, aryl boronic acid-capped fluorophores remain a prevalent strategy in the selective detection of $\mathrm{H}_{2} \mathrm{O}_{2}$. Recent advances include detection of $\mathrm{H}_{2} \mathrm{O}_{2}$ in ischemia-reperfusion injury (20), chemiluminescent detection through a capped luciferin substrate (21), and the use of boronate-capped probes that trigger 1,6-elimination reactions on phenol generation (20).

\section{Phosphinates}

The nucleophilic property of superoxide can also be exploited to cleave phosphinate groups. This strategy was spearheaded by $\mathrm{Xu}$ et al. (22) when they synthesized diphenylphosphinate-capped fluorescein and naphthofluorescein analogs, which were able to detect superoxide down to $0.1 \mathrm{nM}$. These probes are also useful for in vitro imaging, as was illustrated by imaging superoxide formation in macrophages after stimulation with phorbol 12-myristate 13-acetate, an oxidative burst stimulant. This strategy was further expanded on by Zhang et al. (23), who created a NIR dye that allowed for in vivo imaging of superoxide. Recently, this strategy was also expanded to include bioluminescence by Liu et al. (24), who used a phosphinate-capped luciferin to detect polystyrene-induced superoxide formation in cells. Furthermore, a mitochondriatargeting probe (NA-T) that saw a shift in fluorescence after phosphinate deprotection and subsequent 1,6-elimination to release 
an unmodified diketopyrrolopyrrole dye has been developed (25). NA-T showed high specificity toward superoxide-mediated cleavage in the presence of a wide range of ROS and was used for imaging of phorbol 12-myristate 13-acetate/lipopolysaccharide-triggered superoxide formation in Daphnia magna.

\section{TPEF}

TPEF is a type of fluorescence microscopy that uses 2 NIR photons to electronically excite a fluorophore from the ground to the singlet excited state, as opposed to directly exciting the fluorophore with a high-energy light source. Because long wavelengths penetrate more deeply into tissue, the use of TPEF, rather than confocal microscopy, offers many benefits. Aside from deeper tissue penetration, TPEF also offers reduced photobleaching and photodamage, suppressed background signal, and minimized scattering.

Common organic fluorophores, such as fluorescein derivatives, have been modified with ROS-sensitive moieties that quench fluorescence; however, on ROS-mediated cleavage they possess fluorescence suitable for TPEF (26). Furthermore, the team of Lewis recently developed an azulene fluorophore for TPEF bioimaging of RNS and ROS (27). The boronic ester is cleaved in the presence of $\mathrm{H}_{2} \mathrm{O}_{2}$ and $\mathrm{ONOO}^{-}$, and because of strong electrondonating groups the azulene derivative undergoes an intramolecular charge transfer that shifts the absorption and emission of the polyaromatic hydrocarbon to the visible range. Because there has been extensive study of an immense list of chromophores, which absorb in the visible spectra, modifying current chromophores for TPEF has been a popular choice.

\section{ULTRASOUND IMAGING}

Ultrasound imaging, or sonography, is a technique that uses highfrequency sound waves for real-time in vivo imaging. Currently, microbubbles are one of the most used ultrasound contrast agents for clinical ultrasound imaging. However, for imaging ROS, there are limitations to using microbubbles as ultrasound contrast agents.

\section{Microbubbles}

Microbubbles vary in size from 1 to $10 \mu \mathrm{m}$ in diameter. Conventional microbubbles are composed of lipids, polymers, and surfactants, among others, which encapsulate a gas. However, by chemically modifying hydrazine, $\mathrm{N}_{2}$ encapsulation as an organic compound is possible. The Murthy lab has previously demonstrated the use of chemically generated microbubbles to image in vivo oxidative stress via ultrasound (28). Allylhydrazine encapsulated within a liposome proved efficient in detecting $10 \mathrm{mM}$ ROS in vitro and in vivo (28). In the presence of ROS, the amine from allylhydrazine oxidizes into 2-propenyl-diazene and thus allows for a retro-ene reaction that results in the release of nitrogen and propylene gas. This increase in gas concentration creates acoustic impedance that can be detected acoustically.

\section{Photoacoustic Imaging Using Nanoparticles}

When tissue absorbs light and converts it to heat via vibrational relaxation, thermoelastic expansion may result and then generate ultrasound waves. This is known as the photoacoustic effect. Previously, researchers have used polymers and nanoparticles as a nanoplatform for developing photoacoustic probes (29). In vivo ratiometric photoacoustic imaging is a relatively new method that has been used to monitor ROS and RNS (29,30). When a

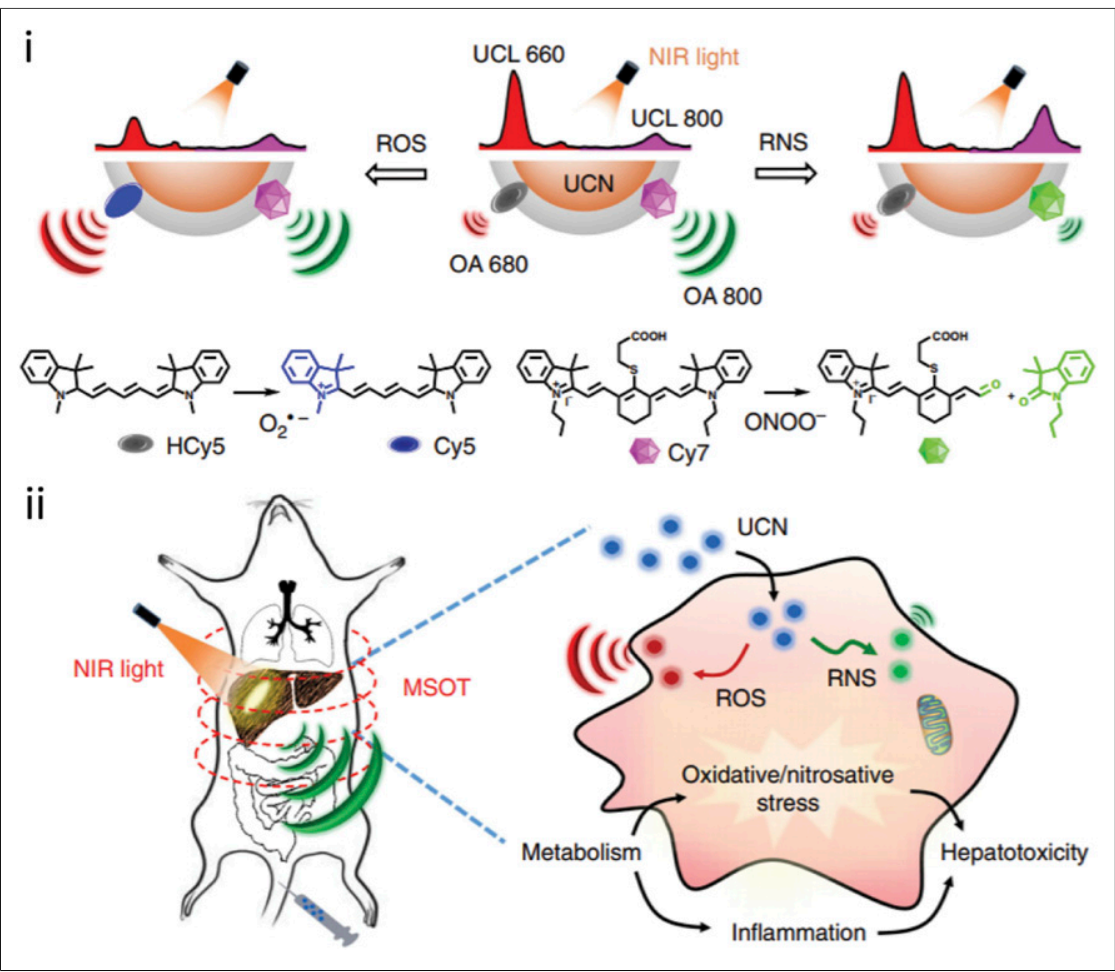

FIGURE 2. (i) Illustration of HCy5 (ROS) and Cy7 (RNS) on upconversion nanocrystal (UCN) surface. In presence of ROS, nonfluorescent HCy5 is converted into fluorescent Cy5, whereas in presence of RNS, fluorescent Cy7 decomposes, causing signal reduction at $800 \mathrm{~nm}$. (ii) Schematic representation of UCN in live mice for bioimaging. MSQT = multispectral optoacoustic tomography; $\mathrm{UCL}=$ upconverted luminescence. (Reprinted from Ai et al. (32).) nanoparticle is coupled with an ROS-sensitive dye, the nanoparticle emits an optoacoustic signal. In the presence of ROS, absorption or fluorescence of the chromophore is blueshifted because of degradation whereas absorption or fluorescence of the nanoparticle remains unaffected. Overlaying the images when monitoring at NIR versus visible wavelength generates a representative image.

Using nanoparticles to image and generate ROS in cancer cells is of great importance for theranostics. By incorporating multifunctional groups on semiconducting perylene diimide, Chen and team developed a theranostic nanoparticle, perylene diimideIR790s-iron/cisplatin (31), for ratiometric photoacoustic imaging. IR790s, a cyanine derivative, absorbs light at $790 \mathrm{~nm}$ but decomposes in the presence of ROS, losing its absorptivity. The change in absorption ratio between perylene diimide and IR790s allows for ratiometric imaging. The cisplatin prodrug activates nicotinamide adenine dinucleotide phosphate oxidase, thus forming superoxides followed by $\mathrm{H}_{2} \mathrm{O}_{2}$ generation by superoxide dismutase. $\mathrm{Fe}^{3+}$ then converts $\mathrm{H}_{2} \mathrm{O}_{2}$ into hydroxyl radicals via the Fenton reaction, which then reacts with IR790s to quench absorption. This technique to induce 
ROS for cancer therapy also allows for real-time monitoring during treatment.

\section{Photoacoustic Imaging Using Upconversion Nanocrystals}

Detecting multiple radical species independently is invaluable in ROS imaging. By incorporating 2 cyanine dyes-hydro-Cy5 and $\mathrm{Cy} 7$, which are sensitive to 2 specific reactive species-onto the surface of an upconversion nanoprobe, Xing and team were able to screen the presence of ROS and RNS via multispectral optoacoustic tomography by ratiometrically monitoring multiple radicals (32). Upconversion nanocrystals have been used because of their antiStokes-shift properties. Unlike TPEF, upconversion nanocrystals rely on sequential, rather than simultaneous, absorption of photons. Therefore, it is possible to excite the nanocrystal with NIR light and observe emission in the visible spectra (33). Because emission of the nanocrystal overlaps the absorption of the cyanine dye, individual monitoring of the cyanine dye is possible. In the presence of ROS there is a fluorescence increase and, thus, an increase in optoacoustic signal. Conversely, in the presence of RNS there is a decrease in fluorescence and, thus, a decrease in the optoacoustic signal (Fig. 2).

\section{LUMINESCENCE}

ROS imaging usually requires a light source to photoexcite a fluorophore, thus resulting in photoluminescence. However, there are processes that result in spontaneous light emission without photoexcitation, known as chemiluminescence and bioluminescence. Although chemiluminescence involves a chemical process, bioluminescence involves the use of luciferase enzymes to oxidize luciferin to oxyluciferin, thus producing luminescence. Luminescence microscopy minimizes background emission and thus provides better signal-tonoise ratios and eliminates phototoxicity and photobleaching.

\section{Chemiluminescence}

Unlike fluorophores, chemiluminescent probes do not autofluoresce, thus making them desirable for bioimaging. However, some drawbacks are a short emission time and short wavelengths. To address the issue of short emission time, Ren and team synthesized a chemiluminescent polymer dot probe (hemin-Pdots) that relies on chemiluminescence resonance energy transfer to enhance the chemiluminescence lifetime to about $10 \mathrm{~h}$ in the presence of an L-012, a luminol analog, and $\mathrm{H}_{2} \mathrm{O}_{2}$ (34). In the presence of $\mathrm{H}_{2} \mathrm{O}_{2}, \mathrm{~L}-012$ oxidizes, and chemiluminescence that is 100 times greater than that of luminol is observed (34). Slow diffusion of the oxidized L-012 through the hemin-Pdots nanoparticles increases the chemiluminescence lifetime via chemiluminescence resonance energy transfer, where the luminol is the energy donor and the hemin-Pdot is the acceptor. Indeed, the slow diffusion dynamics of such chemiluminescent systems show a 700-fold enhanced chemiluminescence over $10 \mathrm{~h}$.

One of the problems in ROS imaging via luminescence is correlating the readout to ROS concentration. Contag and team have previously described use of a reporter probe, coelenterazine, to measure superoxide dynamics (35). Interestingly, they were able to observe a change in chemiluminescence during cellular respiration, as well as in response to variant glucose concentrations. By generating an in vitro baseline using coelenterazine, their system shows promise not only for in vivo imaging but also for quantification of superoxide concentrations.

\section{CONCLUSION}

ROS continues to play a central role in biology and medicine, and there is great interest in imaging their concentration in cell cultures and in vivo. Although significant progress has been made toward imaging ROS, several challenges remain. In particular, although a wide variety of turn-on probes have been developed that can indicate the presence of ROS in tissues and in cells, their analysis is always made indirectly, via comparison against control cells. A central goal in the field of ROS imaging, which still has not been achieved, is the development of ROS probes that can quantitatively measure the concentration of ROS in cells, similar in function to calcium chelating probes. Ratiometric ROS probes provide a significant step toward this goal but are still unable to provide true quantitation. In addition, ROS probes that can measure tissue ROS concentrations via PET, MRI, or ultrasound imaging are also greatly needed because these imaging modalities are widely used in the clinic. Most of the existing ROS probes are based on fluorescent imaging, and fundamentally new chemical strategies for imaging ROS have to be developed to detect ROS via PET, MRI, and ultrasound. The field of ROS imaging has made remarkable progress in the last $10 \mathrm{y}$, and we anticipate that there will be many exciting new developments in the years ahead.

\section{DISCLOSURE}

This work was supported by the National Institutes of Health (grants R01EB023776, R01AI117064, R33AI119115, and R01EB020008) and the Innovative Genomics Institute. No other potential conflict of interest relevant to this article was reported.

\section{REFERENCES}

1. Hensley K, Robinson KA, Gabbita SP, Salsman S, Floyd RA. Reactive oxygen species, cell signaling, and cell injury. Free Radic Biol Med. 2000;28:1456-1462.

2. Pizzino G, Irrera N, Cucinotta M, et al. Oxidative stress: harms and benefits for human health. Oxid Med Cell Longev. 2017;2017:8416763.

3. Andreae WA. A sensitive method for the estimation of hydrogen peroxide in biological materials. Nature. 1955;175:859-860.

4. Kundu K, Knight SF, Willett N, Lee S, Taylor WR, Murthy N. Hydrocyanines: a class of fluorescent sensors that can image reactive oxygen species in cell culture, tissue, and in vivo. Angew Chem Int Ed Engl. 2009;48:299-303.

5. Sadlowski CM, Maity S, Kundu K, Murthy N. Hydrocyanines: a versatile family of probes for imaging radical oxidants in vitro and in vivo. Mol Syst Des Eng. 2017;2:191-200.

6. Maity S, Das S, Sadlowski CM, Zhang J, Vegesna GK, Murthy N. Thiophene bridged hydrocyanine: a new fluorogenic ROS probe. Chem Commun (Camb). 2017;53:10184-10187.

7. Al-Karmi S, Albu SA, Vito A, et al. Preparation of an ${ }^{18} \mathrm{~F}$-labeled hydrocyanine dye as a multimodal probe for reactive oxygen species. Chem Eur J. 2017;23:254-258.

8. Andina D, Brambilla D, Munzinger N, et al. Development of a modular ratiometric fluorescent probe for the detection of extracellular superoxide. Chem Eur J. 2017;23:4765-4769.

9. Zhang L, Meng X, Shan X, et al. Integrin $\alpha v \beta 3$-specific hydrocyanine for cooperative targeting of glioblastoma with high sensitivity and specificity. Anal Chem. 2019;91:12587-12595.

10. Gomes A, Fernandes E, Lima JLFC. Fluorescence probes used for detection of reactive oxygen species. J Biochem Biophys Methods. 2005;65:45-80.

11. Zhang H, Liu J, Liu C, et al. Imaging lysosomal highly reactive oxygen species and lighting up cancer cells and tumors enabled by a Si-rhodamine-based nearinfrared fluorescent probe. Biomaterials. 2017;133:60-69.

12. Wang L, Liu J, Zhao S, et al. Fluorescence imaging of hypochlorous acid and peroxynitrite in vitro and in vivo with emission wavelength beyond $750 \mathrm{~nm}$. Chem Commun (Camb). 2020;56:7718-7721.

13. Maeda H, Fukuyasu Y, Yoshida S, et al. Fluorescent probes for hydrogen peroxide based on a non-oxidative mechanism. Angew Chem Int Ed Engl. 2004;43:2389-2391.

14. Maeda H, Yamamoto K, Nomura Y, et al. A design of fluorescent probes for superoxide based on a nonredox mechanism. J Am Chem Soc. 2005;127:68-69.

15. Hu JJ, Wong N-K, Ye S, et al. Fluorescent probe HKSOX-1 for imaging and detection of endogenous superoxide in live cells and in vivo. $J$ Am Chem Soc. 2015;137:6837-6843. 
16. Han X, Wang R, Song X, Yu F, Lv C, Chen L. A mitochondrial-targeting nearinfrared fluorescent probe for bioimaging and evaluating endogenous superoxide anion changes during ischemia/reperfusion injury. Biomaterials. 2018;156:134-146.

17. Lu D, Zhou L, Wang R, et al. A two-photon fluorescent probe for endogenous superoxide anion radical detection and imaging in living cells and tissues. Sens Actuators B Chem. 2017;250:259-266.

18. Peiró Cadahía J, Previtali V, Troelsen NS, Clausen MH. Prodrug strategies for targeted therapy triggered by reactive oxygen species. MedChemComm. 2019;10:1531-1549.

19. Chang MCY, Pralle A, Isacoff EY, Chang CJ. A selective, cell-permeable optical probe for hydrogen peroxide in living cells. J Am Chem Soc. 2004;126:15392-15393.

20. Xu R, Wang Y, You H, Zhang L, Wang Y, Chen L. A near-infrared fluorescent probe for evaluating endogenous hydrogen peroxide during ischemia/reperfusion injury. Analyst. 2019;144:2556-2564.

21. Zielonka J, Podsiadły R, Zielonka M, Hardy M, Kalyanaraman B. On the use of peroxy-caged luciferin (PCL-1) probe for bioluminescent detection of inflammatory oxidants in vitro and in vivo: identification of reaction intermediates and oxidant-specific minor products. Free Radic Biol Med. 2016;99:32-42.

22. Xu K, Liu X, Tang B, Yang G, Yang Y, An L. Design of a phosphinate-based fluorescent probe for superoxide detection in mouse peritoneal macrophages. Chem Eur J. 2007;13:1411-1416.

23. Zhang J, Li C, Zhang R, et al. A phosphinate-based near-infrared fluorescence probe for imaging the superoxide radical anion in vitro and in vivo. Chem Commun (Camb). 2016;52:2679-2682.

24. Liu X, Tian X, Xu X, Lu J. Design of a phosphinate-based bioluminescent probe for superoxide radical anion imaging in living cells. Luminescence. 2018;33:1101-1106.

25. Zhang Z, Fan J, Zhao Y, Kang Y, Du J, Peng X. Mitochondria-accessing ratiometric fluorescent probe for imaging endogenous superoxide anion in live cells and Daphnia magna. ACS Sens. 2018;3:735-741.
26. Tsakanova G, Arakelova E, Ayvazyan V, et al. Two-photon microscopy imaging of oxidative stress in human living erythrocytes. Biomed Opt Express, BOE. 2017;8:5834-5846.

27. Murfin LC, Weber M, Park SJ, et al. Azulene-derived fluorescent probe for bioimaging: detection of reactive oxygen and nitrogen species by two-photon microscopy. J Am Chem Soc. 2019;141:19389-19396.

28. Perng JK, Lee S, Kundu K, et al. Ultrasound imaging of oxidative stress in vivo with chemically-generated gas microbubbles. Ann Biomed Eng. 2012;40: 2059-2068.

29. Pu K, Shuhendler AJ, Jokerst JV, et al. Semiconducting polymer nanoparticles as photoacoustic molecular imaging probes in living mice. Nat Nanotechnol. 2014;9:233-239.

30. Zheng J, Zeng Q, Zhang R, Xing D, Zhang T. Dynamic-reversible photoacoustic probe for continuous ratiometric sensing and imaging of redox status in vivo. J Am Chem Soc. 2019;141:19226-19230.

31. Yang Z, Dai Y, Yin C, et al. Activatable semiconducting theranostics: simultaneous generation and ratiometric photoacoustic imaging of reactive oxygen species in vivo. Adv Mater. 2018;30:e1707509.

32. Ai X, Wang Z, Cheong $\mathrm{H}$, et al. Multispectral optoacoustic imaging of dynamic redox correlation and pathophysiological progression utilizing upconversion nanoprobes. Nat Commun. 2019;10:1087.

33. Zhou B, Shi B, Jin D, Liu X. Controlling upconversion nanocrystals for emerging applications. Nat Nanotechnol. 2015;10:924-936.

34. Cai L, Deng L, Huang X, Ren J. Catalytic chemiluminescence polymer dots for ultrasensitive in vivo imaging of intrinsic reactive oxygen species in mice. Anal Chem. 2018;90:6929-6935.

35. Bronsart LL, Stokes C, Contag CH. Chemiluminescence imaging of superoxide anion detects beta-cell function and mass. PLoS One. 2016;11:e0146601. 